\title{
Il corpo nelle esperienze di disastro e attivismo in siti contaminati dall'amianto
}

The body in the experiences of disaster and activism in asbestos-contaminated sites

Agata Mazzeo

\section{(2) OpenEdition}

\section{Journals}

Edizione digitale

URL: http://journals.openedition.org/aam/2667

DOI: $10.4000 /$ aam. 2667

ISSN: 2038-3215

\section{Editore}

Dipartimento Culture e Società - Università di Palermo

Notizia bibliografica digitale

Agata Mazzeo, «ll corpo nelle esperienze di disastro e attivismo in siti contaminati dall'amianto»,

Archivio antropologico mediterraneo [Online], Anno XXIII, n. 22 (1) | 2020, online dal 20 juin 2020 consultato il 30 janvier 2021. URL: http://journals.openedition.org/aam/2667 ; DOI: https://doi.org/ 10.4000/aam.2667

Questo documento è stato generato automaticamente il 30 janvier 2021.

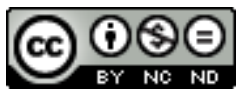

Archivio antropologico mediterraneo è distribuita con Licenza Creative Commons Attribuzione - Non commerciale - Non opere derivate 4.0 Internazionale. 


\section{Il corpo nelle esperienze di disastro e attivismo in siti contaminati dall'amianto}

The body in the experiences of disaster and activism in asbestos-contaminated sites

Agata Mazzeo

\section{Introduzione}

1 L'amianto, dal latino amiantus "incorruttibile", è un termine generico che indica un insieme di minerali accomunati dal fatto di essere costituiti da fibre resistenti al fuoco e facilmente manipolabili. Nel corso del XX secolo, l'uso dell'amianto si è diffuso globalmente e ha interessato i più disparati settori industriali (ad esempio, industria edilizia, ferroviaria, automobilistica e aeroportuale) (Rossi 2008; Roselli 2010). Il brevetto Eternit, ovvero una composizione di cemento e amianto, fu inventato dal chimico austriaco Hatschek nel 1902 e fu poi venduto rapidamente in tutto il mondo (Roselli 2010). Le esperienze di disastro e attivismo discusse in questo articolo sono legate a storie di lavoro ed esposizione presso due cementifici di prodotti a marchio Eternit, con sede a Casale Monferrato (Italia) e Osasco (Brasile).

2 Il boom economico dell'amianto sul mercato globale è durato per quasi tutto il secolo scorso nonostante, fin dalla seconda metà degli anni Sessanta del Novecento, circolassero fra la comunità scientifica internazionale i risultati delle prime indagini epidemiologiche che mostravano la relazione fra l'esposizione all'amianto $\mathrm{e}$ l'insorgenza di un cancro particolare, il mesotelioma maligno (MM) (Selikoff et al. 1965). Ad oggi si stima che più di 107.000 persone muoiano ogni anno nel mondo a causa di malattie amianto-correlate (AC) (Prüss-Ustün et al. 2011) e che più di 125 milioni di persone siano ancora esposte all'amianto nei luoghi di lavoro (WHO 2018). L'Agenzia Internazionale $\mathrm{di}$ Ricerca sul Cancro riconosce come malattie provocate 
dall'esposizione all'amianto l'asbestosi, il cancro della laringe, dell'esofago, dell'ovaio, del polmone e il MM (IARC 2012).

In Italia, più di 27.000 diagnosi di MM sono state registrate fino al 2015 dal Registro Nazionale Mesoteliomi-ReNaM (INAIL 2018). In Brasile, invece, quasi 1.200 casi di malattia AC sono stati registrati fra il 2007 e il 2013 (Ministério da Saúde 2016). Tuttavia, il numero di diagnosi di malattie AC si considera sottostimato nel contesto brasiliano. Un simile fenomeno di sottodiagnosi è frequente nei paesi dove l'uso dell'amianto è ancora consentito, come dibattuto da studi condotti da gruppi di ricerca transdisciplinari e internazionali (Pasetto et al. 2014; Marsili et al. 2016). Ad oggi, 67 paesi hanno proibito qualsiasi uso dell'amianto sui loro territori (Kazan-Allen 2019a).

In questo articolo l'impatto del mercato dell'amianto sulla salute pubblica è indicato in termini di disastro. In base ad una definizione antropologica, un disastro è un processo e mai un evento isolato, per quanto improvvise possano essere alcune sue manifestazioni (cfr. Oliver-Smith, Hoffman 1999; Hoffman, Oliver-Smith 2002; Ligi 2009, 2011a, 2011b; Benadusi et al. 2011; Benadusi 2015). Nel caso dei disastri provocati dal mercato tossico dell'amianto, il luogo entro cui questi disastri occorrono e devastano è innanzitutto il corpo di chi ha respirato la "polvere", come spesso le fibre d'amianto sono indicate proprio da chi quella polvere l'ha "mangiata", altra espressione frequentemente usata dagli interlocutori incontrati sul campo per indicare l'inalazione delle cancerogene fibre.

5 Antropologi che hanno condotto le loro ricerche in siti contaminati da amianto hanno richiamato l'attenzione sul "silenzio" che avvolge tali disastri (Petrillo 2015), sulla “invisibilità" dell'epidemia di morti e malattie AC (Braun, Kisting 2006; Braun 2008) e sulla "elusività" dell'asbesto (Waldman 2011). Personalmente, ho qualificato tali processi di disastro come "invisibili" in quanto attraversati da dinamiche transnazionali che, nei diversi contesti socioculturali, hanno celato il pericolo derivante dall'esposizione all'amianto e velato d'incertezza le cause delle malattie AC, determinando il mancato riconoscimento, a livello giuridico, economico e biomedico, dell'ingiustizia sociale e della sofferenza vissute dagli esposti e dai sopravvissuti (Mazzeo 2017).

6 Nella lettura che propongo i disastri provocati dal mercato tossico dell'amianto si declinano a) nelle esperienze di malattie AC; b) nelle esperienze di contaminazione e del conseguente rischio di sviluppare una malattia $\mathrm{AC}$, in particolare il MM, un cancro alla cui diagnosi l'aspettativa di vita è in media di nove mesi contro un periodo di latenza che può superare i 40 anni (INAIL 2018); c) nelle esperienze di sofferenza legate alla perdita di un proprio caro e al vivere (sopravvivere) in siti contaminati dove vi è un'elevata mortalità AC. Attraverso tali esperienze, gli attori sociali coinvolti vivono quei processi di incorporazione attraverso cui le dinamiche transnazionali del mercato dell'amianto pervadono e sconvolgono l'abituale essere-nel-mondo degli esposti e di chi soffre a causa di un'esposizione, propria o altrui.

7 Tramite il linguaggio e le pratiche dell'attivismo, le fibre d'amianto, annidate nei corpi degli esposti, possono essere lette e riconosciute come le prove tangibili di un'ingiustizia subita, di un processo di disastro ancora in corso. A partire dall'esperienza (corporea) del disastro, coloro coinvolti in un simile processo, possono mettere in atto pratiche ed elaborare significati nuovi attraverso cui reinventare il loro essere e agire nel mondo, mobilitandosi per ottenere il riconoscimento della propria sofferenza in nome di obiettivi di giustizia sociale. 
8 La riflessione proposta mira a sottolineare la necessità di considerare la dimensione sociale e corporea dei processi di disastro così come dei processi di produzione di saperi nel momento in cui si affronta una questione di salute pubblica globale come quella rappresentata dai disastri AC. La sofferenza vissuta da e attraverso il corpo contaminato può mettere in crisi pratiche e discorsi che, prima dell'irruzione violenta del disastro nei corpi e nelle vite degli esposti, rappresentavano la normalità del proprio essere-nel-mondo. Il corpo che soffre è attraversato da una carica eversiva, tuttavia, è necessario che tale forza sia riconosciuta e legittimata perché possano essere intrapresi percorsi inediti di azione e riflessione. In tal senso, gli esposti e i sopravvissuti all'amianto hanno trovato nel linguaggio dell'attivismo, come anche in quello biomedico, giuridico e della lotta sindacale, le parole per indirizzare le potenzialità di critica che attraversano le esperienze di sofferenza verso nuovi orizzonti di cura e lotta.

\section{Contesti di ricerca e note metodologiche}

9 I dati discussi in questo contributo sono emersi da una ricerca etnografica multisituata condotta fra l'Italia e il Brasile, iniziata nel 2008 e non ancora conclusasi. La ricerca ha interessato, in particolare, tre contesti italiani (Bari, Casale Monferrato e Bologna) e uno brasiliano (Osasco, nello stato di São Paulo). L'approccio multisituato e globale (cfr. Marcus 1995; Buroway et al. 2000) ai quattro contesti di ricerca ha permesso di collocare le esperienze quotidiane di disastro e attivismo indagate localmente nell'intricato intreccio delle dinamiche in gioco nel mercato globale dell'amianto e del movimento per la proibizione dell'amianto nel mondo. Data l'impossibilità di contestualizzare e analizzare con il dovuto approfondimento dati provenienti da tutti i contesti finora indagati qui, si è scelto di soffermarsi sul contesto italiano di Casale Monferrato e quello brasiliano di Osasco, legati da connessioni storiche, economiche e sociali. Tali connessioni hanno rappresentato le traiettorie lungo le quali mi sono mossa durante la ricerca. Per un approfondimento sugli altri contesti da me indagati rimando ad altre pubblicazioni (Mazzeo 2014, 2017, 2018a, 2018b, 2018c).

10 Presso lo stabilimento Eternit di Casale Monferrato, aperto nel 1906, le attività cessarono nel 1986. Nel corso di ottant'anni, circa 5.000 lavoratori vi trovarono impiego (Rossi 2008). Lo stabilimento è stato demolito nel 2007; nell'area bonificata è stato costruito un parco, inaugurato nel settembre 2016 con il nome "Parco EterNot".

11 Agli inizi degli anni Ottanta, un gruppo di operai dell'Eternit, insospettito dal numero delle morti fra colleghi e dalle proprie cattive condizioni di salute, iniziò a cercare interlocutori presso locali istituzioni di salute pubblica e organizzazioni sindacali per definire il malessere che li affliggeva e rivendicare migliori condizioni di lavoro. Si stavano compiendo i primi passi di una mobilitazione dal basso che, negli anni, ha visto aggregarsi cittadini ed ex-lavoratori in una battaglia civile che ha avuto una risonanza molto forte a livello non soltanto nazionale, ma anche internazionale. Tale mobilitazione portò, ad esempio, nel 1987, il comune di Casale Monferrato, primo in Italia, a proibire l'uso dell'asbesto (Mossano 2010).

12 A livello internazionale, grande eco ha avuto il processo giudiziario che la locale associazione anti-amianto, l'Associazione Familiari e Vittime Amianto (AFeVA), ha contribuito a istituire contro la multinazionale Eternit, per la prima volta sotto accusa per il crimine di disastro ambientale doloso. Sebbene in ultima istanza il crimine non 
sia stato riconosciuto perché ritenuto prescritto, il cosiddetto "processo Eternit" ha avuto un impatto notevole sul movimento per la proibizione globale dell'amianto (Rossi 2012). Personalmente, ho avuto modo di seguire l'eco che esso ha avuto sull'attivismo anti-amianto in Brasile, dove è situato il secondo contesto di ricerca cui faccio riferimento.

Ad Osasco, una città di circa 700.000 abitanti, confinante con la parte occidentale della megalopoli di São Paulo, lo stabilimento "Eternit do Brasil Cimento e Amianto S/A" fu aperto nel 1941. Esso ha rappresentato il più esteso stabilimento a marchio Eternit in America Latina. La produzione cessò nel 1993 e l'intero sito industriale fu demolito nel 1995. Il movimento anti-amianto brasiliano mosse i suoi primi passi proprio nell'Osasco di quegli anni con la fondazione dell'Associazione Brasiliana Esposti Amianto (ABREA), divenuta poi il punto di riferimento per il movimento anti-amianto nell'intero Paese.

In Italia e Brasile, l'estrazione, la lavorazione, la commercializzazione e la regolamentazione dell'amianto, fino alla sua totale proibizione in entrambi i paesi, hanno seguito tempi diversi. In particolare, mi soffermo sulle discrepanze temporali riguardanti l'inizio della lavorazione dell'amianto e la proibizione di qualsiasi uso del minerale nonché di prodotti che lo contengano. In Italia, sede della più grande miniera d'amianto nell'area geografica europea, la lavorazione industriale dell'amianto ebbe inizio nel primo decennio del Novecento (Iocca 2011), mentre in Brasile, sia l'estrazione sia la lavorazione dell'amianto iniziarono fra la fine degli anni Trenta e gli inizi degli anni Quaranta del Novecento (Giannasi 2012). Questa discrepanza ha condizionato un movimento, particolarmente rilevante nel secondo dopoguerra, di capitali e corpi dall'area europea a quella latinoamericana, dove vennero importate, adattandole ai contesti locali, politiche gestionali e strategie di (non) comunicazione del rischio già sperimentate in Europa.

Entrambi i paesi hanno giocato un ruolo cruciale nel mercato globale dell'amianto. In Italia, fra il 1945 e il 1992 (anno della proibizione) si sono estratti quasi quattro milioni di tonnellate di amianto, mentre, nello stesso intervallo di tempo, se ne sono importate quasi due milioni (INAIL 2018). Il Brasile, d'altra parte, ha rappresentato il terzo paese produttore di amianto al mondo (US Geological Survey 2017), con più di quattro milioni e mezzo di tonnellate di amianto prodotte nel XX secolo (Iocca 2011), fino alla messa al bando del minerale su tutto il territorio nazionale per decisione del Supremo Tribunale Federale, alla fine del 2017. L'attuazione della messa al bando, tuttavia, è stata ritardata fino al 2019 e rappresenta, tutt'oggi, motivo di aspro conflitto fra il locale movimento anti-amianto e le parti private interessate a portare avanti l'estrazione del minerale, in particolare ai fini dell'esportazione della materia prima verso il mercato asiatico (Kazan-Allen 2019b).

16 A Casale Monferrato ho condotto una ricerca sul campo di due mesi nel 2012, avendo potuto approcciare gli attivisti AFeVA grazie ai contatti avviati in occasione di una precedente ricerca condotta nel 2009 a Bari, mia città natale. Negli anni, il mio posizionamento nei confronti degli argomenti di studio, così come nelle relazioni con $\mathrm{i}$ miei interlocutori sul campo, è cambiato. Il ruolo riconosciutomi dai miei interlocutori brasiliani, i quali, nel 2015, avevano potuto vedere in me una ricercatrice da anni inequivocabilmente impegnata a sostegno dell'attivismo anti-amianto, ha di fatto reso possibile la realizzazione della ricerca stessa e definito in maniera netta la mia posizione nei confronti di una questione particolarmente delicata nel contesto brasiliano. Pur nella consapevolezza che qualsiasi sforzo conoscitivo sia 
inesorabilmente determinato da scelte per le quali si è responsabili sia sul piano etico sia sul piano scientifico, la dimensione pubblica e impegnata della mia ricerca, in certe occasioni, è stata più marcata di quanto avrei mai potuto immaginare. Rimando ad altri miei scritti per una riflessione più accurata sulle dinamiche e le problematiche affrontate durante il personale coinvolgimento nello studio dei disastri e dell'attivismo amianto-correlati (Mazzeo 2013, 2014). La centralità del corpo nei processi di disastro così come nelle pratiche di attivismo ambisce a rappresentare il filo conduttore fra $\mathrm{i}$ dati etnografici presentati, nella consapevolezza che ogni contesto di ricerca presenta delle proprie specificità. I dati discussi di seguito offrono esempi di come la conoscenza e il passato incorporati dai lavoratori esposti siano stati cruciali per l'elaborazione di un'evidenza epidemiologica dell'impatto dell'esposizione all'amianto sulla salute pubblica e di come il corpo che soffre, mettendo in discussione il "normale" essere-nelmondo, conservi un'intrinseca forza eversiva anche quando, a causa di un'irreversibile assenza, si trova ad essere evocato, esibito e ricordato.

In entrambi i contesti i miei principali interlocutori sul campo sono stati ex-lavoratori esposti all'amianto presso gli stabilimenti Eternit di Casale Monferrato e Osasco. Ho intervistato anche familiari di lavoratori deceduti. Tutti i miei interlocutori sono stati attivisti o comunque simpatizzanti della lotta anti-amianto. I dati che seguono si riferiscono ad esperienze di disastro legate a storie di esposizione professionale all'amianto, nonostante nel corso della mia ricerca mi sia soffermata anche su esperienze di disastro legate a esposizioni ambientali (Mazzeo 2014, 2017, 2018b).

\section{Il corpo nelle esperienze del disastro}

18 Il corpo che soffre rappresenta un problema, concentra su di sé pensieri, discorsi e pratiche (Good 1999: 191). Allo stesso tempo, diviene detonatore di nuovi saperi, scatena la riflessione, rivela la storicità del "naturale" e smaschera i processi di causazione alla base di presunte "fatalità". Le citazioni riportate in questa sezione sono frammenti di interviste condotte con ex-lavoratori Eternit presso gli stabilimenti di Casale Monferrato e Osasco. I nomi con i quali mi riferisco loro sono pseudonimi. Giuseppe, 66 anni nel 2012, con una diagnosi di asbestosi al 42\%, ha lavorato all'Eternit dal 1966 al 1986, nel reparto, particolarmente polveroso, "materie prime", dove venivano smistati i sacchi d'amianto.

Dei trenta operai che eravamo, siamo rimasti in tre. Lì c'era tutta quella polvere che ci siamo mangiati.

Mi fa paura morire per quell'affare dell'amianto, ci sono amici miei, del reparto dov'ero io, che sono morti e che ancora adesso si ammalano e non vado nemmeno a trovarli all'ospedale... so che devo fare quella fine anch'io [...]. Qualche mese fa ho fatto una lastra, ho due affari come due dischi nei polmoni, il mio dottore mi ha spiegato tutto... bel ricordo ti ha lasciato l'Eternit, speriamo non si muovano.

Antonio, 75 anni nel 2012, con una diagnosi di asbestosi al 50\%, ha lavorato all'Eternit di Casale Monferrato dal 1960 fino alla chiusura, nel 1986.

Si lavorava proprio con la nebbia, ma la gente non lo sapeva che [l'amianto] era nocivo, sapeva che effettivamente a tanti la respirazione diventava affannosa, poi non si poteva proprio più respirare, era sufficiente una broncopolmonite che ci lasciavi la pelle perché morivi soffocato, però non sapevano ancora del male più terribile che era il mesotelioma... questi aghi [le fibre d'amianto] che si piantano nei polmoni sollecitano le cellule cancerogene... 
Con l'asbestosi, i polmoni si cementificano. L'asbestosi è un problema, ma quello più grave è rappresentato dal mesotelioma [...].

Si sapeva che si moriva soffocati, tant'è vero che ad un certo punto hanno vietato $i$ manifesti funebri, per non creare allarmismi... C'era, infatti, l'abitudine che quando moriva uno dell'Eternit si metteva il manifesto vicino alla portineria centrale così gli altri potevano andare alla sepoltura, al funerale, poi un bel momento, con delle scuse che non ricordo più quali fossero, non hanno più permesso che si mettessero dei manifesti... la realtà era che non volevano creare allarmismo, sapevano benissimo quello che [l'amianto] provocava... [...]

I familiari di mia moglie sono morti in tre, tutti per mesotelioma [...]. Io ho problemi di respirazione, non posso fare sforzi, ma sto bene... però... ho sempre l'incubo di fare la morte che hanno fatto mia suocera e i miei cognati, l'incubo di una morte così terribile...

Nel 2015, ad Osasco (Brasile) ho intervistato Chico, 79 anni, il quale aveva lavorato all'Eternit di Osasco dal 1952 al 1985. Agli inizi degli anni Novanta, Chico ha ricevuto una diagnosi di asbestosi e placche pleuriche.

Gradualmente le placche s'ingrandiscono e la capacità dei tuoi polmoni diminuisce [...] Ho sempre questa mancanza di respiro e sono consapevole che ci sono cose che non posso fare più. Cerco di non pensarci troppo perché è inutile pensarci. Pensarci mi fa sentire triste e arrabbiato. Pensare non aiuta. Devo imparare a vivere col danno che ho subito.

21 Sempre ad Osasco nel 2015, ho intervistato Beatriz, 71 anni, attivista ABREA. Beatriz ha perso suo marito Carlos, nel 2008, all'età di 66 anni, a causa del MM. Solo dopo la morte di Carlos, Beatriz ha iniziato a partecipare alla vita dell'associazione. Durante la nostra intervista, Beatriz ha descritto il suo coinvolgimento nell'attivismo come un modo per ringraziare l'ABREA e ricambiare l'affetto e il supporto ricevuti da Carlos, spiegandomi come la proibizione dell'amianto sia necessaria per prevenire che altre famiglie come la sua vivano simili esperienze di sofferenza. Carlos aveva lavorato all'Eternit di Osasco dal 1964 al 1968, nel settore amministrativo. Tramite l'ABREA, di cui aveva iniziato a far parte prima di ricevere la diagnosi di malattia AC, Carlos aveva imparato molte cose sul MM, informandosi e vedendo molti suoi compagni morire. Alla vista del referto radiografico, anticipando il medico, Carlos enunciò egli stesso la diagnosi del suo mesotelioma. All'epoca della mia ricerca sul campo, il lutto senza fine di Beatriz e dei compagni rendeva Carlos un punto di riferimento importante nella lotta portata avanti quotidianamente dall'ABREA, come discuterò nella prossima sezione.

L'impegno nelle locali associazioni anti-amianto ha dato ai miei interlocutori le parole per nominare e curare la propria sofferenza, attraverso il linguaggio biomedico, il riconoscimento giuridico di un crimine e i percorsi di cura che essi stessi avevano e stavano ancora contribuendo a costruire, reinventare e ampliare, dando concretezza a ideali di giustizia sociale nella vita quotidiana degli afflitti. Ad esempio, al momento della mia ricerca sul campo nel 2012, gli attivisti AFeVA erano coinvolti in una trentennale mobilitazione resa ancora più frenetica dall'allora in corso "processo Eternit". Attraverso attività come l'organizzazione di campagne di sensibilizzazione, convegni e assemblee pubbliche, essi si riconoscevano come sopravvissuti ad un unico disastro e formavano una comunità fra le cui reti intessere percorsi di cura per una sofferenza tanto intima e privata quanto sociale e collettiva (Mazzeo 2018c), avendo il mercato dell'amianto provocato, su una popolazione di circa 35.000 abitanti, quasi 2.000 vittime (Rossi 2012). 
23 Ad Osasco, lo scenario urbano presentava delle dinamiche economiche e socioculturali profondamente diverse da quelle riscontrabili nel contesto casalese. Per gli attivisti ABREA il senso di appartenenza ad una comunità di sopravvissuti si sostanziava nelle pratiche volte ad assicurare agli esposti, innanzitutto, l'accesso a cure mediche. La cura della sofferenza AC si concretizzava nel non abbandono (sociale ed economico) delle famiglie coinvolte direttamente nelle esperienze di disastro, in una città come Osasco dove, ai confini di una megalopoli attraversata da un costante flusso di capitali e lavoratori alla ricerca di condizioni di vita migliori, in un paese attraversato da violente disuguaglianze sociali, i legami di comunità assumevano il significato di preziose, ma fragili, risorse da creare e salvaguardare.

\section{Il corpo nelle esperienze di attivismo}

24 Nel considerare il corpo come luogo e agente di memoria, saperi e lotte intendo riconoscere dei margini di azione all'attore sociale, il quale incorpora e allo stesso tempo si fa agente di processi storici e socio-culturali (Bourdieu 1972; Bourdieu, Wacquant 1992). Nei termini dell'attivismo, i processi che caratterizzano il mercato globale dell'amianto sono letti come processi attraverso cui sono stati commessi "crimini d'impresa" subdoli e protratti nel tempo (Altopiedi 2011), ai danni di "innocenti", così come molti dei miei interlocutori definivano se stessi e i propri cari. Dall'analisi dei dati emersi dalle ricerche condotte in Italia e Brasile, il corpo non è mai apparso come mero oggetto, passivo campo di battaglia su cui agiscono forze distruttive. Il corpo, invece, è apparso anche, e simultaneamente, come il luogo in cui a quelle stesse forze distruttive si oppone resistenza, come lo scenario a partire dal quale le tracce del passato innescano pratiche di memoria e conoscenza che assumono la valenza di pratiche di lotta e cura (Mazzeo 2018c).

Come enuncia Thomas Csordas, è opportuno tenere sotto controllo i rischi derivanti dall'adottare la "metafora famelica" del corpo come testo, riconoscendo, invece, al corpo la capacità di produrre significati (Csordas 2003). Attraverso il paradigma dell'incorporazione, il corpo esposto, il corpo che porta dentro di sé le fibre d'amianto, non è un mero "serbatoio" delle prove di un'avvenuta esposizione, o solo un "testo", da imparare a leggere attraverso i linguaggi dell'attivismo, del sapere biomedico o giuridico. Custode delle tracce del passato, il corpo ha un ruolo attivo nel produrre significati nuovi e attraverso di esso processi transnazionali invisibili si sostanziano nell'esperienza e si rendono manifesti (Scheper-Hughes 1994; Fassin 2007, 2008). D'altra parte, l'esperienza di sofferenza, mettendo in crisi l'abituale essere-nel-mondo ha un effetto detonatore di nuove pratiche e nuovi significati. Il corpo che soffre perturba e insinua domande sul significato del proprio dolore e della propria esistenza (Kleinman 2006; Wilkinson, Kleinman 2016); tali domande possono aprire prospettive inedite nell'ambito della cura, della cittadinanza e delle forme istituzionalizzate di sapere. Un'esperienza di malattia come il MM, ancora oggi considerato inguaribile nei termini biomedici di una definitiva eradicazione del cancro, non solo mette in crisi il mondo di chi soffre, ma, svelando i limiti di un approccio biomedico spesso miope dinanzi alla dimensione socio-culturale di qualsiasi patologia, può rappresentare la condizione da cui ripensare i significati di "cura" e considerare i processi attraverso i quali le stesse evidenze scientifiche di una problematica di salute pubblica vengono elaborate. L'evidenza stessa del disastro AC si è iniziata a produrre quando i lavoratori esposti 
hanno messo a disposizione dello sguardo biomedico il proprio corpo contaminato e sofferente.

A Casale Monferrato, un primo studio che rivelò l'entità dell'impatto della lavorazione dell'amianto sulla realtà locale fu realizzato da professionisti della salute pubblica delle locali istituzioni, interpellati da lavoratori e cittadini. Lo studio, basato sull'analisi di 70 cartelle cliniche di pazienti ai quali, tra il 1973 e il 1983, era stato diagnosticato il MM e di cui 61 erano residenti a Casale Monferrato e nove presso cittadine limitrofe, rivelarono un dato inaspettato: la maggior parte dei MM diagnosticati era da porre in relazione con un'esposizione ambientale, e non professionale, all'amianto (Mossano 2010). Questo primo studio fu seguito da un secondo, denominato "Progetto Cemento Amianto", affidato ad un gruppo di ricerca costituito da oncologi, pneumologi, medici del lavoro e epidemiologi (cfr. Magnani et al. 1987). I risultati emersi da queste prime indagini legittimarono la mobilitazione civile casalese e portarono il Comune a proibire, nel 1987, l'uso dell'amianto, nonostante il ruolo cruciale che la produzione dell'amianto aveva nel tessuto socio-economico locale.

Per quanto riguarda il contesto brasiliano, invece, cito come esempio una delle prime pubblicazioni che ha rappresentato l'avvio della costruzione dell'evidenza epidemiologica dell'impatto del mercato dell'amianto sulla salute pubblica in Brasile. L'articolo, pubblicato nel $2001 \mathrm{e}$ basato su uno studio che aveva visto la partecipazione volontaria di 868 lavoratori esposti all'amianto (la maggior parte dei quali exdipendenti presso lo stabilimento Eternit di Osasco e attivisti ABREA), riconosce esplicitamente il ruolo avuto dagli ex-lavoratori, divenuti attivisti, nell'aver permesso la realizzazione dello studio (Algranti et al. 2001).

Il corpo diviene centrale nelle pratiche di attivismo nella misura in cui il corpo contaminato viene usato come uno strumento per rivendicare il riconoscimento di un'ingiustizia subita. Nuove pratiche di cittadinanza vengono messe in atto a partire da una "condizione biosociale" condivisa (Rabinow 1992) e strategie di mobilitazione, ricollegabili a "lotte per il riconoscimento" (Hobson 2003), vengono ad essere elaborate proprio a partire dal corpo che soffre (Brown et al. 2004; Brown, Zavestoski 2004).

29 L'antropologa Adriana Petryna (2002), indagando le connessioni fra il processo di costruzione del sapere scientifico e la gestione politica del post-disastro di Černobyl', ha riflettuto su come le norme che regolano la cittadinanza e eventi catastrofici siano strettamente intrecciate e divulghino sapere scientifico, giuridico e biomedico. In un contesto storico assai delicato come quello che stava attraversano l'Ucraina nel 1986, con il collasso dell'Unione Sovietica e il passaggio drastico ad un'economia di mercato e ad un sistema politico fragile, le pratiche di sopravvivenza poste in essere dai sopravvissuti al disastro erano finalizzate ad ottenere un riconoscimento ufficiale del loro essere stati esposti alle radiazioni. Ruotando attorno a tale riconoscimento tutta una serie di benefici altrimenti inaccessibili nonostante le reali condizioni di necessità, appariva necessario ricostruire un legame con il disastro per rendere riconoscibili e riconosciuti i danni provocati dal disastro stesso.

Per una definizione di giustizia e in particolare di giustizia sociale nell'ambito della salute pubblica globale mi riferisco in particolare alle riflessioni proposte da Hofrichter (1993, 2003). In linea con Fraser (2007), la definizione di giustizia a cui si rifà l'analisi delle pratiche di attivismo qui proposta considera la giustizia come quell'insieme complesso di dinamiche che permettono a tutti di prendere parte alla vita sociale da pari. In questo senso, gli attivisti anti-amianto incontrati in Italia e Brasile stavano 
dando concretezza a ideali di giustizia nella loro vita quotidiana entrando, da pari e in quanto corpi sofferenti, in quei processi di negoziazione di saperi e diritti aventi un impatto diretto sulle loro vite.

31 Infine, nelle pratiche di attivismo anti-amianto, il corpo contaminato continua ad essere uno strumento di azione politica anche quando viene a mancare, nel momento in cui è ricordato e rievocato e la sua immagine continua ad essere esibita, divenendo simbolo allo stesso tempo di lacerazione profonda vissuta nel corpo e negli affetti, ma anche di una strenua resistenza al decadimento fisico e alla violenza di processi subdoli di disastro. A tale proposito, voglio concludere questa sezione con la citazione di un'intervista condotta con Beatriz la quale, nei seguenti termini, commentava la decisione del marito, Carlos, di farsi fotografare nel suo letto d'ospedale, pochi giorni prima di morire di MM.

Penso che quella foto sia stata scattata da qualcuno dell'ABREA andato a visitarlo all'ospedale. Penso che fu proprio mio marito a chiedere di essere fotografato e ciò è molto triste per me. Io non ho quella foto in casa. Immagino che con quella foto lui volesse mostrare al mondo quanto sia pericoloso l'amianto e che proibirlo corrisponda ad una questione di vita o di morte. Mio marito si dedicava molto all'ABREA e egli stesso chiese che la sua foto fosse esposta ad ogni occasione possibile, così adesso, quando c'è un evento $o$ in occasione delle campagne di sensibilizzazione sul rischio amianto, i suoi compagni dell'ABREA espongono sempre quella foto. Io non posso impedirlo, anche se per me è molto doloroso $\mathrm{e}$ difficile vedere ogni volta quell'immagine. Non posso chiedere che non venga mostrata, sarebbe un atto di codardia da parte mia perché se lui ha chiesto di essere fotografato e mostrare al mondo intero la sua immagine, io non posso oppormi e dire che non voglio, per questo ho dato il mio consenso a che l'ABREA possa fare libero uso di quell'immagine, in questo modo, mi ripeto, la sua volontà è fatta.

L'esposizione del ritratto di Carlos in occasione di eventi pubblici e campagne di sensibilizzazione sui danni dell'esposizione all'amianto porta il lutto di Beatriz, ogni volta rinnovato, al di là della sfera privata e della ristretta cerchia affettiva. Per le comunità di sopravvissuti ai disastri $\mathrm{AC}$, le pratiche di lutto diventano pratiche di cura e di lotta. Nelle parole di Zhang, «il rifiuto di porre fine al lutto diviene un'azione politica di protesta contro l'ingiustizia sociale responsabile della perdita della vita» (2012: 271). Simili pratiche messe in atto dalla mobilitazione anti-amianto, sono pratiche di cura nella misura in cui i sopravvissuti possono elaborare significati per la propria sofferenza nelle relazioni affettive e nelle occasioni d'incontro che il coinvolgimento nell'attivismo offre loro. Esse sono, allo stesso tempo, pratiche di lotta in quanto permettono a coloro colpiti dall'impatto del mercato dell'amianto sulle proprie vite, di mettere in relazione la propria sofferenza con specifici processi di disastro e, in ragione di una simile consapevolezza, mobilitarsi per il riconoscimento dell'ingiustizia subita.

\section{Conclusioni}

Tramite le parole e le pratiche dell'attivismo, coloro che sono coinvolti nelle esperienze di disastro $\mathrm{AC}$ possono situare in una condivisa storia di ingiustizia e lotta le proprie esperienze di sofferenza che, in questo modo, travalicano la sfera privata e possono acquisire significati nuovi. In tal senso, le pratiche di attivismo sono pratiche di cura per una sofferenza che può permanere non riconosciuta, come i processi di disastro che 
la provocano, e attraverso la partecipazione, il ricordo di chi muore e il non abbandono di chi sopravvive, chi soffre può prendersi cura di sé e degli altri.

Il corpo contaminato dei lavoratori esposti all'amianto, incontrati in Italia e Brasile, custodiva le tracce di un passato che era ancora presente. Mantenendone dolorosamente viva la memoria, il corpo degli esposti legittima le lotte intraprese in nome della giustizia sociale, essendo, allo stesso tempo, il luogo del disastro, ma anche strumento di riscatto, il punto di partenza per rivendicare l'accesso a diritti negati e costruirne di nuovi.

L'analisi delle dinamiche in gioco nei processi di disastro e attivismo AC rivela in maniera paradigmatica la dimensione sociale di qualsiasi esperienza di sofferenza, evidenza scientifica e proposta di cura. Non è semplice individuare il legame fra ordine biologico e ordine sociale, gli intrecci tra sfera sociale e sfera privata, soprattutto quando tali legami corrono silenziosi nel corpo degli esposti e sono velati da un'invisibilità altrettanto socialmente costruita. Attraverso l'attivismo, i sopravvissuti ai disastri AC hanno portato alla luce tali intrecci e hanno potuto individuare i legami fra la propria sofferenza e precisi processi storici e socio-economici.

In entrambi i contesti di ricerca, la mobilitazione dal basso è emersa a partire dall'esperienza, condivisa da un numero gradualmente crescente di persone che avevano iniziato a contarsi, riconoscersi e organizzarsi sulla base di una sofferenza che inizialmente era apparsa inspiegabile, non comunicabile e per questo non condivisibile e non riconosciuta. Il coinvolgimento nella lotta anti-amianto, rappresentando per coloro che vi avevano preso parte la possibilità di nominare la propria sofferenza in termini di ingiustizia e disastro, ha rappresentato l'occasione di entrare, con la capacità di negoziare significati, in quelle dinamiche attraverso le quali l'impatto del mercato dell'amianto sulla salute pubblica è stato reso visibile e strategie per affrontarlo, arginarlo e contrastarlo sono state elaborate e messe in atto.

\section{BIBLIOGRAFIA}

Algranti E. et al., 2001 «Non-Malignant Asbestos Related Diseases in Brazilian Asbestos Cement Workers», in American Journal of Industrial Medicine, 40: 240-254.

Altopiedi R., 2011 Un Caso di Criminalità d'Impresa. L'Eternit di Casale Monferrato, L'Harmattan, Torino.

Benadusi M., 2015 «Antropologia dei Disastri. Ricerca, Attivismo, Applicazione. Un'Introduzione», in Antropologia dei disastri. Ricerca, Attivismo, Applicazione (Special Issue), Antropologia Pubblica, 1(1): 33-60.

Benadusi M. et al. (eds.), 2011 Disasters, Development and Humanitarian Aid. New Challenges for Anthropology, Guaraldi, Rimini.

Bourdieu P., 1972 Esquisse d'une théorie de la pratique. Précédé de trois études d'ethnologie kabyle, Editions du Seuil, Paris. 
Bourdieu P., Wacquant L. J., 1992 An Invitation to Reflexive Sociology, University of Chicago Press, Chicago.

Braun L., 2008 «Structuring Silence: Asbestos and Biomedical Research in Britain and South Africa», in Race \& Class, 50(1): 59-78.

Braun L., Kisting S., 2006 «Asbestos-Related Disease in South Africa. The Social Production of an Invisible Epidemic», in American Journal of Public Health, 96(8): 2-12.

Brown P., Zavestoski S., 2004 «Social Movements in Health: An Introduction», in Sociology of Health \& Illness, 26(6): 679-694.

Brown P. et al., 2004 «Embodied Health Movements: Uncharted Territory in Social Movement Research», in Sociology of Health \& Illness 26(1): 1-31.

Csordas T. J., 1990 «Embodiment as a Paradigm for Anthropology», in Ethos, 18(1): 5-47.

Csordas T. J., 2003 «Incorporazione e fenomenologia culturale, in Antropologia, 3(3): 19-42.

Fassin D., 2007 When Bodies Remember. Experiences and Politics of AIDS in South Africa. University of California Press, Berkeley.

Fassin D., 2008 «The Embodied Past», in Social Anthropology/Anthropologie Sociale, 16(3): 312-328.

Fraser N., 2007 «Re-framing Justice in a Globalizing World», in T. Lovell (ed.), (Mis)Recognition, Social Inequality and Social Justice: Nancy Fraser and Pierre Bourdieu, Routledge, New York: 17-35.

Giannasi F., 2012 «Eternit in Brazil», in Allen D., Kazan-Allen L. (eds.), Eternit and the Great Asbestos Trial, Justasec Print Services Ltd., London: 65-71.

Good B. J., 1999 Narrare la Malattia. Lo Sguardo Antropologico sul Rapporto Medico-Paziente, Edizioni di Comunità, Torino.

Hobson B., 2003 Recognition Struggles and Social Movements. Cultural Claims, Contested Identities, Power and Agency, Cambridge University Press, Cambridge.

Hoffman S. M., Oliver-Smith A. (eds.), 2002 Catastrophe \& Culture. The Anthropology of Disaster, School of American Research Press, Santa Fe.

Hofrichter R., 1993 Toxic Struggles: The Theory and Practice of Environmental Justice, New Society Publishers, Philadelphia.

Hofrichter R., 2003 (ed.) Health and Social Justice. Politics, Ideology, and Inequity in the Distribution of Disease, Jossey-Bass, San Francisco.

IARC-International Agency for Research on Cancer, 2012 «Asbestos (chrysotile, mosite, crocidolite, tremolite, actinolite, and anthophyllite)», in IARC (ed.), Metals, Arsenic, Dusts, and Fibres. A Review of Human Carcinogens (Monograph on the Evaluation of Carcinogenic Risks to Humans, 100C), IARC, Lyon: 219-309.

INAIL-Istituto nazionale Assicurazione Infortuni sul Lavoro, 2018 Il Registro Nazionale dei Mesoteliomi. VI Rapporto, Tipolitografia Inail, Milano.

Iocca G., 2011 Casale Monferrato: la Polvere che Uccide. Voci dalla Chernobyl Italiana, Ediesse, Roma.

Kazan-Allen L., 2019a Current Asbestos Bans, http://ibasecretariat.org/alpha_ban_list.php (visualizzato il 12/04/2020).

Kazan-Allen L., 2019b Report from Asian Ban Asbestos Mission to Brazil, April 2019, http:// ibasecretariat.org/lka-status-report-from-asian-ban-asbestos-mission-to-brazil-apr-24-2019.php (visualizzato il 12/04/2020). 
Kleinman A., 2006 What Really Matters. Living a Moral Life Amidst Uncertainty and Danger, Oxford University Press, New York.

Ligi G., 2009 Antropologia dei Disastri, Laterza, Roma-Bari.

Ligi G., 2011a «Valori Culturali del Paesaggio e Antropologia dei Disastri», in La Ricerca Folklorica, 64: 119-129.

Ligi G., 2011b «Emotions and Knowledge in the Anthropology of Disasters», in Benadusi M. et al. (eds.), Disasters, Development, and Humanitarian Aid. New Challenges for Anthropology, Guaraldi, Rimini: 47-64.

Magnani C. et al., 1987 «Modalità per Tumori ed Altre Malattie del Sistema Respiratorio tra i Lavoratori del Cemento-Amianto a Casale Monferrato. Uno Studio di Coorte Storico», in Medicina del Lavoro 78: 411-453.

Marcus G. E., 1995 «Ethnography in/of the World System: The Emergence of Multi-Sited Ethnography», in Annual Review of Anthropology, 24: 95-117.

Marsili D. et al., 2016 «Prevention of Asbestos-Related Disease in Countries Currently Using Asbestos», in International Journal of Environmental Research and Public Health 13(494): 1-19.

Mazzeo A., 2013 «(When) To Be Engaged is Inescapable», in The Innovia Foundation Newsletter, 21: 8-12.

Mazzeo A., 2014 «Contaminazione, Coinvolgimento, Impegno. Riflessioni su una ricerca di antropologia medica in contesti urbani affetti da disastri ambientali e sociali provocati dalla lavorazione del cemento-amianto in Italia», in Palmisano A. L. (a cura di), Antropologia Applicata, Pensa Editore, San Cesario di Lecce: $217-250$.

Mazzeo A., 2017 «Disastri Invisibili e Pratiche di Attivismo», in Antropologia, 4(1): 203-219.

Mazzeo A., 2018a «The Temporalities of Asbestos Mining and Community Activism», in The Extractive Industries and Society, 5: 223-229.

Mazzeo A., 2018b «L'Impatto dell'Esposizione all'Amianto su una Comunità di Lavoratori e Residenti in Italia e Brasile», in Minoia C., Comba P. (a cura di), Amianto: Un Fantasma del Passato o una Storia Infinita?, New Press Edizioni, Cermenate: 467-481.

Mazzeo A., 2018c «“Care-Activism” in Asbestos Contaminated Communities», in Quaranta I. et al. (eds.), Assemblages, Transformations, and the Politics of Care, Bologna University Press, Bologna: 93-111.

Mossano S., 2010 Malapolvere. Una Città si Ribella ai Signori dell'Amianto, Edizioni Sonda, Casale Monferrato.

Ministério da Saúde, 2016 «Doenças Relacionadas ao Asbesto na Saúde do Trabalhador, Brasil, 2007-2013», in Boletim Epidemiológico 47(4): 1-8.

Oliver-Smith A., Hoffman S. M. (eds.), 1999 The Angry Hearth. Disaster in Anthropological Perspective, Routledge, New York.

Pasetto R. et al., 2014 «Occupational Burden of Asbestos-related Cancer in Argentina, Brazil, Colombia, and Mexico», in Icahn School of Medicine at Mount Sinai. Annals of Global Health, 80: 263-268.

Petrillo A. (a cura di), 2015 Il Silenzio della Polvere Capitale, Verità e Morte in una Storia Meridionale di Amianto, Mimesis Edizioni, Milano-Udine. 
Petryna A., 2002 Life Exposed: Biological Citizens After Chernobyl, Princeton University Press, Princeton.

Prüss-Ustün A. et al., 2011 «Knowns and Unknowns on Burden of Disease due to Chemicals: A Systematic Review», in Environmental Health, 10(9): 1-15.

Rabinow P., 1992 «Studies in the Anthropology of Reason», in Anthropology Today 8(5): 7-10.

Roselli M., 2010 La Mentira del Amianto. Fortunas y Delitos, Ediciones del Genal, Málaga.

Rossi G., 2008 La Lana della Salamandra. La Vera Storia della Strage dell'Amianto a Casale Monferrato, Ediesse, Roma.

Rossi G., 2012 Amianto. Processo alle Fabbriche della Morte, Melampo, Milano.

Scheper-Hughes N., 1994 «Embodied Knowledge: Thinking with the Body in Critical Medical Anthropology», in Borofsky R. (ed.), Assessing Cultural Anthropology, MacGraw-Hill, New York: 229-242.

Selikoff I. J. et al., 1965 «Relation between Exposure to Asbestos and Mesothelioma», in New England Journal of Medicine, 272(11): 560-565.

US Geological Survey, 2017 Mineral Commodity Summaries available on line, https:// minerals.usgs.gov/minerals/pubs/commodity/asbestos/ (visualizzato il 12/04/2020).

Waldman L., 2011 The Politics of Asbestos. Understanding of Risk, Disease, and Protest, Earthscan, London.

WHO-World Health Organization, 2018 Asbestos: Elimination of Asbestos-Related Diseases, Fact sheet, 15 February 2018.

Wilkinson I., Kleinman A., 2016 A Passion for Society. How we Think about Human Suffering, University of California Press, Berkeley.

Zhang E. Y., 2012 «Mourning», in D. Fassin (ed.), A Companion to Moral Anthropology, WileyBlackwell, Malden: 264-282.

\section{RIASSUNTI}

In questo articolo si propone una riflessione sulla centralità del corpo nelle esperienze di disastro e attivismo in due siti contaminati dall'amianto, in Italia e Brasile. I disastri amianto-correlati si manifestano e disvelano il proprio impatto innanzitutto attraverso il corpo degli esposti nel momento in cui, rendendosi manifesti i sintomi di malattie contratte a seguito dell'inalazione delle cancerogene fibre che costituiscono i minerali d'amianto, va in crisi il "naturale", sociale e corporeo, essere-nel-mondo. Il corpo contaminato, tuttavia, non è soltanto il luogo in cui il disastro si manifesta con tutta la sua violenza, ma è anche il luogo della memoria, nella misura in cui esso custodisce le tracce di tali disastri, che spesso permangono invisibili. Attraverso il linguaggio e le pratiche dell'attivismo, il corpo viene ad agire in quanto strumento di conoscenza e lotta. A partire dalla sofferenza incorporata vengono elaborati saperi nuovi e messe in atto pratiche che danno concretezza, nella vita quotidiana degli esposti e dei sopravvissuti, a obiettivi di giustizia sociale.

This article focuses on the centrality of the body in the experiences of disaster and activism in two asbestos-contaminated sites in Italy and Brazil. Asbestos-related disasters appear and reveal their impact primarily through the body of the exposed person who experiences the symptoms of the diseases caused by the inhalation of the carcinogenic fibres of asbestos minerals. Then, the 
"natural", social and corporeal, being-in-the-world of a person is upset. However, the contaminated body is not only the place where the disaster becomes violently evident, but it is also the place of a memory since it preserves the traces of such disasters, which often remain invisible. The body acts as a tool of knowledge and struggle through the language and the practices of activism. From the embodied suffering then a new knowledge and new practices emerge that give concreteness to objectives of social justice, in the daily life of the exposed and survivors.

\section{INDICE}

Keywords : body, disaster, asbestos, activism, multi-situated ethnography Parole chiave : corpo, disastro, amianto, attivismo, etnografia multisituata

\section{AUTORE}

AGATA MAZZEO

Dipartimento di Storia, Culture, Civiltà, Università di Bologna agata.mazzeo2@unibo.it 